\title{
Waking Genioglossal Electromyogram in Sleep Apnea Patients versus Normal Controls (a Neuromuscular Compensatory Mechanism)
}

William S. Mezzanotte, Douglas J. Tangel, and David P. White

Pulmonary Division, Denver Veterans Administration Medical Center, National Jewish Center, and University of Colorado Health

Sciences Center, Denver, Colorado 80220

\begin{abstract}
Pharyngeal collapse in obstructive sleep apnea patients is likely a product of a sleep-related decrement in pharyngeal dilator muscle activity superimposed upon abnormal airway anatomy. We postulate that during wakefulness, increased pharyngeal dilator muscle activity in apnea patients compensates for diminished airway size thus maintaining patency. We studied the waking genioglossus (GG) electromyogram (EMG) activity in 11 OSA patients and 14 age-matched controls to determine if GG activity is higher in the awake state in apnea patients than controls. To make this determination, we developed a reproducible methodology whereby true maximal GG EMG could be defined and thus basal activity quantitated as a percentage of this maximal value. Therefore, direct comparisons of basal activity between individuals was possible. We observed apnea patients to have significantly greater basal genioglossal activity compared to controls $(40.6 \pm 5.6 \%$ vs. $12.7 \pm 1.7 \%$ of maximum). This difference persisted when size-matched subsets were compared. This augmented GG activity in apnea patients could be reduced with positive airway pressure. We speculate that this neuromuscular compensation present during wakefulness in apnea patients may be lost during sleep leading to airway collapse. (J. Clin. Invest. 1992.89:1571-1579.) Key words: continuous positive airway pressure $\bullet$ pharyngeal dilator muscle $\bullet$ upper airway
\end{abstract}

\section{Introduction}

The precise explanation for pharyngeal collapse during sleep in patients with obstructive sleep apnea (OSA) ${ }^{1}$ remains unclear. Most investigators (1-4) would argue it to be a product of a sleep-induced fall in upper airway dilator muscle activity occurring in an individual with an anatomically small pharyngeal airway. Although the preponderance of data supports the concept that sleep apnea patients do have reduced pharyngeal airway size $(5-7)$, this is frequently difficult to document $(8,9)$ inasmuch as these patients have little apparent difficulty maintaining adequate airway patency during wakefulness. In addition, the data addressing the influence of sleep on pharyngeal

Address reprint requests to Dr. Mezzanotte, Respiratory Care, 111A, Denver VA Medical Center, 1055 Clermont Street, Denver, CO 80220. Received for publication $13 \mathrm{May} 1991$ and in revised form $8 \mathrm{No}$ vember 1991.

1. Abbreviations used in this paper: CPAP, continuous positive airway pressure; EMG, electromyogram; GG, genioglossus; NREM, non-rapid eye movement; OSA, obstructive sleep apnea.

The Journal of Clinical Investigation, Inc.

Volume 89, May 1992, 1571-1579 dilator muscle activity in apnea patients and normal subjects are inconsistent and potentially confusing.

The genioglossus (GG) is the pharyngeal dilator muscle that has been most extensively studied in both apnea patients and normal subjects. This is due to its important anatomical location and function in influencing upper airway patency as well as its ease of instrumentation. At this time, there appears to be a dichotomy between OSA patients and normal subjects regarding the influence of non-rapid eye movement (NREM) sleep on GG activity. A number of investigators $(1,2)$ have demonstrated the genioglossal EMG to fall during NREM sleep in OSA patients. Conversely, current literature $(10,11)$ indicates that the activity of the genioglossus is well maintained in normal individuals while asleep. No adequate explanation for this discrepancy is currently available.

A variety of studies (12-19) in animals and humans indicates that the pharyngeal muscles (including the GG) can respond to changes in the local upper airway milieu. The most potent local stimulus to pharyngeal muscle activity appears to be negative intraluminal airway pressure (12-19), with several authors demonstrating in both animals $(12,13,15,16)$ and humans $(17,19)$ a reflex genioglossal response to negative airway pressure. In an individual with reduced pharyngeal airway size (such as an apnea patient), greater intraluminal negative pressure is generated during inspiration. Theoretically, this increased negative airway pressure could reflexly stimulate upper airway muscle activity, thus dilating the pharyngeal airway and maintaining reasonable levels of airflow resistance during wakefulness.

If such mechanisms are active in humans, apnea patients would be expected to have greater pharyngeal dilator muscle activity present during wakefulness than is encountered in normal subjects. Such neuromuscular compensation would not only maintain airway patency while awake but could potentially make it difficult to discern differences in waking airway luminal size in apnea patients versus controls using standard imaging techniques. With the onset of sleep, these neuromuscular mechanisms in the human upper airway may be lost leading to a decline in muscle activity and pharyngeal collapse in the apnea patient. In normal subjects in whom such compensatory mechanisms are not required to maintain airway patency, little change in muscle activity would be expected during NREM sleep. Thus, this hypothesis would explain both the discrepancy in sleep effect on pharyngeal dilator muscle activity in apnea patients versus normal individuals as well as why apnea patients can maintain airway patency during wakefulness yet fail to do so asleep.

There are considerable data which support the concept that respiratory compensatory mechanisms are lost during sleep. The ability to compensate for inspiratory-resistive loads is diminished during NREM sleep $(20,21)$, the cough reflex is lost while asleep $(22,23)$, and the ventilatory responses to chemical 
stimuli (hypoxia and hypercapnia) are markedly reduced during sleep (24-28). Therefore, it would not be surprising that a local neuromuscular compensatory mechanism active in the pharyngeal airway during wakefulness might also be inhibited during sleep.

To document the validity of this series of hypotheses, it must be demonstrated that $(a)$ apnea patients have greater pharyngeal muscle (genioglossus) activity present during wakefulness than normal controls, $(b)$ the augmented muscle activity in apnea patients can be inhibited if the appropriate stimulus (probably negative intrapharyngeal pressure) is eliminated (yielding levels of muscle activity similar to normals), (c) sleep produces a loss of this neuromuscular compensation in apnea patients again yielding levels of muscle activity similar to controls, and $(d)$ the sleep-related loss of augmented muscle activity in apnea patients correlates temporally with airway collapse. Most of the concepts described above require methodology whereby the electromyogram (EMG), as a measure of muscle activity, can be directly compared between one individual and another. However, such direct comparison between individuals has not been possible before this time. In this paper we (a) describe a reproducible method of comparing GG muscle activity between subjects, $(b)$ demonstrate that during wakefulness OSA patients have significantly increased GG EMG activity when compared to normal subjects, and (c) demonstrate that positive airway pressure inhibits the genioglossal EMG in apnea patients yielding levels similar to those observed in normal controls.

\section{Methods}

We initially studied 11 males with OSA and 11 healthy age-matched controls with no historical evidence of breathing disorders during sleep (Table I). All apnea patients had $>25$ apneas plus hypopneas per hour of sleep but no history of cardiac or pulmonary disease. Each was newly diagnosed with predominantely obstructive events (defined below) and thus was receiving no therapy for the sleep apnea at the time of our evaluation. Our original intent had been to match patients and controls only for age. However, to exclude any influence of obesity (a common trait in apnea patients) on our observations, three size-matched obese controls documented to be free of apnea by polysomnography $(<10$ apneas plus hypopneas per hour of sleep) were subsequently studied. When these individuals were added to our control group, we were able to age and size (body mass index) match five patients to five controls (Table II). Thus, when comparing apnea patients to controls, two comparisons will be made: all patients $(n=11)$ versus all controls $(n=14)$ and age plus size-matched controls $(n=5)$ versus patients $(n=5)$. The protocol had the prior approval of the institution's Clinical Investigation Committee with each subject or patient giving informed consent.

Table I. Subject and Patient Characteristics for Study Populations

\begin{tabular}{lcc}
\hline & $\begin{array}{c}\text { Normal controls } \\
(n=14)\end{array}$ & $\begin{array}{c}\text { OSA patients } \\
(n=11)\end{array}$ \\
\hline $\begin{array}{l}\text { Age }(y r) \\
\text { Body mass index } \\
\left(\mathrm{kg} / \mathrm{m}^{2}\right)\end{array}$ & $43.6 \pm 3.5$ & $43.0 \pm 2.5$ \\
$\begin{array}{l}\text { Apnea-hypopnea index } \\
\text { (events per hour of sleep) }\end{array}$ & $28.0 \pm 1.5$ & $41.6 \pm 2.3^{*}$ \\
\hline
\end{tabular}

$* P<0.05$ vs. controls.
Table II. Subject and Patient Characteristics for Age- and Size-matched Individuals

\begin{tabular}{lcc}
\hline & $\begin{array}{c}\text { Normal controls } \\
(n=5)\end{array}$ & $\begin{array}{c}\text { OSA patients } \\
(n=5)\end{array}$ \\
\hline Age $(y r)$ & $49.8 \pm 5.7$ & $44.2 \pm 3.2$ \\
Body mass index $\left(\mathrm{kg} / \mathrm{m}^{2}\right)$ & $34.7 \pm 1.6$ & $35.9 \pm 1.9$
\end{tabular}

Each apnea patient plus three obese controls underwent standard clinical polysomnography. During these studies, sleep was monitored (Electroencaphalogram [EEG], electro-oculogram [EOG], and EMG) and staged in the usual manner (29). In addition, the following variables were routinely recorded: ( $a$ ) nasal and oral airflow (capnographs), (b) respiratory effort (inductance plethysmograph, Respitrace Systems, Ardsley, NY), (c) arterial oxygen saturation (oximeter, Nellcor, Inc., Hayward, CA), (d) anterior tibialis EMG (leg movements), and $(e)$ the electrocardiogram (ECG). The following definitions were employed in analyzing these studies. An apnea was defined as a 10-s or longer pause in respiration. Obstructive apneas were characterized by persistent respiratory effort throughout the event while central apneas occurred in the absence of respiratory effort. Mixed apneas were grouped with obstructive ones. Hypopneas were defined as a $50 \%$ or greater decrement in the airflow signal with an associated $4 \%$ or greater fall in arterial oxygen saturation and were not differentiated into obstructive or central types.

All subjects and patients reported to the laboratory during the day after fasting for at least $3 \mathrm{~h}$ at which time the equipment necessary to monitor the following variables was attached: $(a)$ supraglottic resistance, $(b)$ genioglossal EMG, $(c)$ minute ventilation $(\dot{\mathrm{V} I})$, and $(d)$ endtidal $\mathrm{CO}_{2}\left(\mathrm{P}_{\mathrm{ET}} \mathrm{CO}_{2}\right)$.

\section{Equipment}

Studies required the use of two masks. One was a tight-fitting nasal continuous positive airway pressure mask (Respironics, Inc., Monroeville, PA) which could be attached to a Respironics blower. This device was able to generate between 0 and $20 \mathrm{~cm} \mathrm{H}_{2} \mathrm{O}$ positive pressure in the mask and was used only for nasal continuous positive airway pressure (CPAP) administration. The other was a sealed face mask with a dead space of $\sim 75-100 \mathrm{ml}$ depending on facial configuration. This mask was fitted with inspiratory and expiratory valves (Hans Rudolph, Inc., Kansas City, $\mathrm{MO}$ ) and sampling sites for pressure and $\mathrm{P}_{\mathrm{ET}} \mathrm{CO}_{2}$ determination. The second mask was used for all other studies.

Inspiratory airflow was measured with a model no. 2 pneumotachometer (Fleisch, Inc., Lausanne, Switzerland) placed in the inspiratory line. The pressure drop across the pneumotachometer was measured with a differential pressure transducer (Validyne Inc., Northridge, CA) with flow calibration using a rotameter. Tidal volume was determined from the integrated flow signal (model 7P10, Grass Instrument Co., Quincy, MA) and calibrated using a 3-liter calibration syringe. End-tidal $\mathrm{PCO}_{2}$ was measured with an Ametek infrared $\mathrm{CO}_{2}$ analyzer (Thermox Instruments, Pittsburgh, PA). Pressure in the mask was determined with a second Validyne transducer and pressure at the epiglottis with a pressure-tipped catheter (Millar Instruments, Houston, TX). Both instruments were calibrated simultaneously using a water manometer. There was no amplitude or phase lag between the three signals described above (flow plus mask and epiglottic pressure) at up to $2 \mathrm{~Hz}$. To measure the strength of the tongue, we utilized a lingual force transducer (Neuro Logic, Inc., Bloomington, IN). This device is a semiconductor strain gauge transducer sensitive to direct compression forces. Tongue force against the central area produces activity that can be linearly recorded as force in kilograms $(30,31)$. A magnetic water manometer (Dwyer Instruments, Michigan City, IN) attached to the face mask was used to measure maximum negative inspiratory pressure. All signals were recorded on a 16 channel, model $78 \mathrm{E}$ polygraph (Grass Instrument $\mathrm{Co}$.) and analyzed manually. 


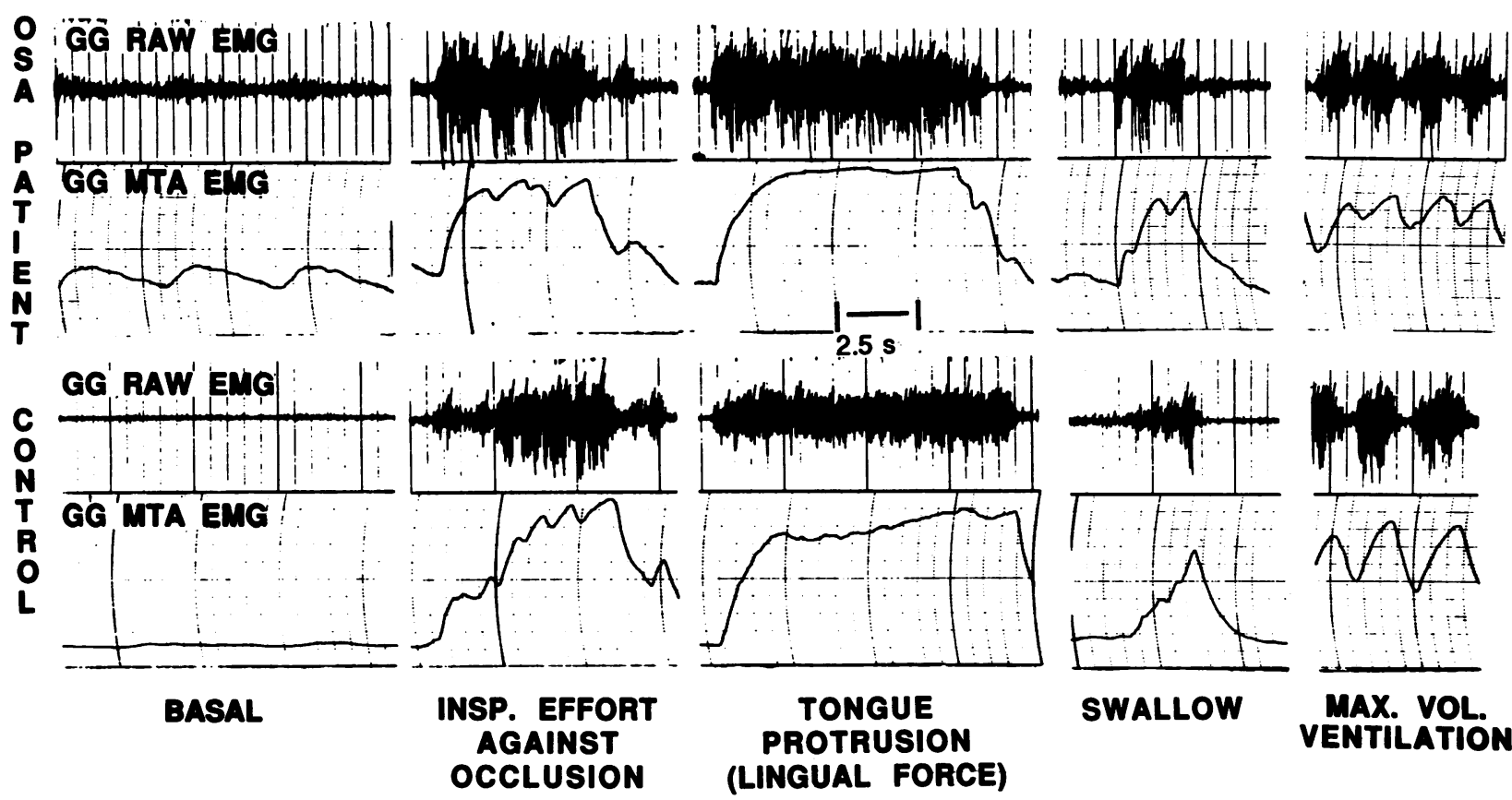

Figure 1. Maneuvers to define maximum GG EMG. Representative raw data from one apnea patient and one normal control demonstrating the maneuvers used to determine maximal genioglossal EMG in all subjects and patients. Each individual has his own scale of EMG activity (from electrical 0 to $100 \%$ ). In addition, this figure demonstrates that basal EMG was a much greater percentage of maximum in the apnea patient than the control.

\section{Techniques}

Supraglottic resistance. The Millar catheter was inserted through an anesthetized (4\% lidocaine), decongested (oxymetazoline HC1) nostril and located at the level of the epiglottis by direct visualization through the mouth. Mask pressure and flow were determine as described above. Supraglottic resistance was calculated as (epiglottic pressure minus mask pressure)/flow and was determined at both peak inspiratory flow and a inspiratory flow rate of 0.2 liters/s.

Genioglossal EMG. Genioglossal EMG was measured by using a pair of unipolar intramuscular electrodes referenced to a single ground thus producing a bipolar recording. Two 25 -gauge needles containing 30-gauge, teflon-coated, stainless steel wires were inserted perorally 2 $\mathrm{cm}$ into the body of the GG muscle at points $3 \mathrm{~mm}$ lateral to the frenulum and midway between the first mandibular incisor and the sublingual fold (3). The needles were quickly removed leaving the wires in place. The EMG signals were amplified, rectified, and integrated by a resistance-capacitance circuit on a moving time average basis with a time constant of $100 \mathrm{~ms}$ (CWE, Inc., Ardmore, PA). Both the raw and moving time average signals were recorded.

To compare the genioglossal EMG between subjects and patients the following methodology was developed. As the actual electrical signal cannot be directly compared between individuals, we attempted to define the true maximal genioglossal EMG using a variety of reproducible, quantifiable maximal respiratory and nonrespiratory maneuvers. Once maximum $(100 \%)$ was defined, the moving time average EMG signal could be scaled between 0 (electrical 0 ) and $100 \%$ (the highest EMG signal encountered during any of the maneuvers described). Thus, basal activity could be measured on this scale and compared between individuals. Maximum genioglossal EMG was produced using a variety of maneuvers which we observed to consistently maximally stimulate this muscle. These maneuvers included: $(a)$ swallow, $(b) 30 \mathrm{~s}$ of maximum voluntary hyperventilation under isocapnia conditions ( $\mathrm{PCO}_{2}$ held constant by the addition of $\mathrm{CO}_{2}$ to the inspiratory line), (c) maximal inspiratory effort against an occluded airway, and $(d)$ maximal forceful protrusion of the tongue against the maxillary alveolar ridge or the lingual force transducer (Fig. 1). The maximal EMG defined by means of these maneuvers is quite different from the "maxi- mal genioglossal EMG" previously described by numerous investigators (32-34). In such studies, the authors defined maximal EMG as the highest EMG value encountered during chemostimulation (usually in response to hypercapnia) and used this value as a reference point in scaling the EMG. We observed that neither hypoxia nor hypercapnia produced a level of muscle activity that even approached that encountered with the volitional maneuvers described above. Because we desired a true maximal signal, these maneuvers were chosen and we believe the maximum EMG was defined.

In most individuals, all maneuvers produced a similar level of GG EMG activity. However, for each subject, the highest EMG signal encountered (defined as $100 \%$ ) was always achieved during either the maximal inspiratory effort against an occluded airway or maximal tongue protrusion. $92 \%$ of the time the tongue protrusion maneuver yielded the highest EMG signal. The mean difference between these two maneuvers (tongue protrusion versus effort against an occluded airway) was $15.4 \pm 3.0 \%$. During these two maneuvers (maximum inspiratory effort against an occluded airway and tongue protrusion) we also measured the effort generated by each individual to insure similar maximal effort between groups. During all occluded inspiratory efforts, we measured the negative inspiratory pressure generated in the mask. During tongue protrusion in four apnea patients and four controls we measured tongue force against the lingual force transducer. The tongue protrusion against the transducer was a similar functional maneuver to the tongue protrusion against the maxillary alveolar ridge and in all eight subjects studied the two maneuvers produced virtually identical levels of GG EMG activity. We found no difference between apnea patients and controls in lingual force (Table III), but there was a small but significant difference in the maximum inspiratory pressure generated with controls generating higher negative inspiratory pressure.

\section{Protocol}

Subjects and patients were studied in a semirecumbent $\left(45^{\circ}\right)$ position in a hospital bed. In order to assure that the hypersomnolent OSA patients were indeed awake during the experiment, gold cup electrodes were placed on their scalp, face, and chin to record standard EEG, 
EOG, and submental EMG signals. Determination of wakefulness was made using standard criteria (29).

Supraglottic resistance was determined before placement of EMG electrodes using the techniques and equipment described above. This resistance was calculated over 20 breaths with mean values being reported. The Millar catheter was then removed, followed by insertion of the genioglossal recording electrodes. Once these electrodes were in place, maximum GG EMG was determined (as described) and the moving time average signal scaled from electrical 0 to $100 \%$ (maximum signal). GG EMG was then recorded during basal unstimulated (nasal) breathing for at least $5 \mathrm{~min}$ and expressed as a percentage of maximum. Finally, the nasal CPAP mask was placed and the GG EMG recorded while 5 and subsequently $10 \mathrm{~cm} \mathrm{H}_{2} \mathrm{O}$ CPAP was applied to the mask. Data were recorded for at least 5 min during each level of CPAP. All equipment was then removed after which the subjects and patients were free to leave.

To assess the reproducibility of our technique, we quantitated genioglossal activity a second time in three apnea patients and three controls several days to several months after their initial study.

\section{Data analysis}

EMG quantitation. The GG EMG signal was scaled as described above and then recorded during quiet, nasal respiration for $5 \mathrm{~min}$. Each breath on every other 30-s epoch (a total of $2.5 \mathrm{~min}$ of recorded data) was subsequently analyzed thus defining the basal EMG. GG moving time average EMG was quantitated as peak phasic and tonic activity in each individual and expressed as a percentage of their own maximum.

During CPAP administration, all signals again were recorded for 5 min at both 5 and $10 \mathrm{~cm} \mathrm{H}_{2} \mathrm{O}$ pressure. Once again, we analyzed every other 30-s epoch over the 5-min period yielding $2.5 \mathrm{~min}$ of meaned data for each CPAP level. GG EMG was again quantitated as peak phasic and tonic activity and expressed as a percentage of the individual's maximal signal.

Airflow resistance. Supraglottic resistance (from above the epiglottis to the external nares) was determined as described above (see subsection Techniques). The resistance was determined at both peak inspiratory flow and at an inspiratory airflow of 0.2 liters/s over 20 consecutive breaths using Millar plus mask pressures and inspiratory flow.

To statistically assess between group (patients versus controls) differences in age, body mass index, GG EMG, airflow resistance, lingual force, and negative inspiratory pressure, unpaired $t$ testing was used. As stated previously, this statistical approach was applied to two samples: all patients versus all controls and age- plus size-matched patients versus controls. To determine the effect of CPAP in each group a parametric two-way analysis of variance and Student-Newman-Keuls multiple comparison test were employed. Correlations between airway resistance and genioglossal activity in apnea patients were made using linear regression analysis. Statistical significance was accepted when $P$ $\leq 0.05$.

To assess the reproducibility of our genioglossal measurement we determined the intraclass correlation coefficient on the six subjects (three apnea patients and three controls) who underwent testing on two

Table III. Effort Determinants from Maximum Genioglossal EMG Assessment

\begin{tabular}{lcr}
\hline & Normal controls & OSA patients \\
\hline $\begin{array}{l}\text { Maximum inspiratory pressure } \\
\left(\mathrm{cm} \mathrm{H}_{2} \mathrm{O}\right)\end{array}$ & $131.6 \pm 5.4$ & $113.5 \pm 5.8^{*}$ \\
$\begin{array}{l}\text { (13 controls, } 10 \text { OSA patients) } \\
\text { Lingual force }(\mathrm{kg})\end{array}$ & $3.1 \pm 0.3$ & $3.9 \pm 0.4$ \\
$(4$ controls, 4 OSA patients) & & \\
\hline
\end{tabular}

${ }^{*} P<0.05$ vs. controls.

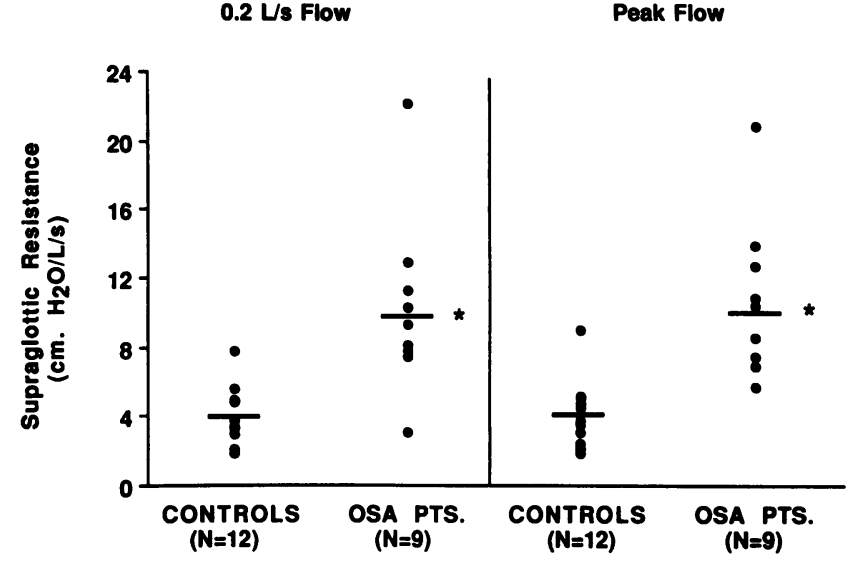

Figure 2. Supraglottic resistance at peak flow and 0.2 liter/s flow. Cumulative data from all subjects and patients demonstrating that apnea patients have a higher mean supraglottic resistance compared to normal controls. ${ }^{*} P<0.05$ vs. controls.

occasions. The intraclass correlation coefficient is a ratio of the between sample variability to the sum of the between sample and within sample variability. The interclass correlation coefficient to ranges from 0 to 1 with 1 being perfect agreement and $>0.75$ considered very good to excellent agreement (35).

\section{Results}

Adequate signals were obtained in all participants with phasic inspiratory genioglossal activity being encountered in all subjects and patients. However, in two control and two apnea patients, technical problems prevented measurement of supraglottic resistance. In one of these same apnea patients, artifact precluded our ability to measure GG EMG during nasal CPAP administration. Representative raw data from one apnea patient and one normal control demonstrating both the basal EMG and the maneuvers used to determine maximal genioglossal EMG are shown in Fig. 1.

Supraglottic resistance. As can be seen in Fig. 2, mean supraglottic resistance at both peak flow and at 0.2 liter/s flow was greater in the apnea patients $(10.8 \pm 1.5$ and $10.2 \pm 1.7 \mathrm{~cm}$ $\mathrm{H}_{2} \mathrm{O}$ per liter/s) compared to controls $(4.3 \pm 0.6$ and $4.0 \pm 0.5 \mathrm{~cm}$ $\mathrm{H}_{2} \mathrm{O}$ per liter/s) although there was considerable overlap between groups.

GG EMG activity during quiet nasal breathing. As can be seen in Fig. 3, the basal peak phasic GG activity of apnea patients represents a significantly higher percentage of maximum activity when compared to controls $(40.6 \pm 5.6 \%$ vs. $12.7 \pm 1.7 \%$ of maximum) although there was some overlap between groups. Fig. 4 demonstrates that both peak phasic and tonic genioglossal activity are significantly different between these two groups with apnea patients demonstrating substantially greater relative muscle activity.

A linear regression analysis was performed assessing the relationship between supraglottic resistance and basal peak phasic GG EMG activity (percentage of maximum) in all participants (patients and controls). A significant correlation was found ( $r=0.48, P=0.03)$ between these variables indicating greater muscle activity in individuals with higher resistance (Fig. 5).

GG EMG activity in age- and size-matched individuals. The GG EMG was compared between apnea patients and con- 


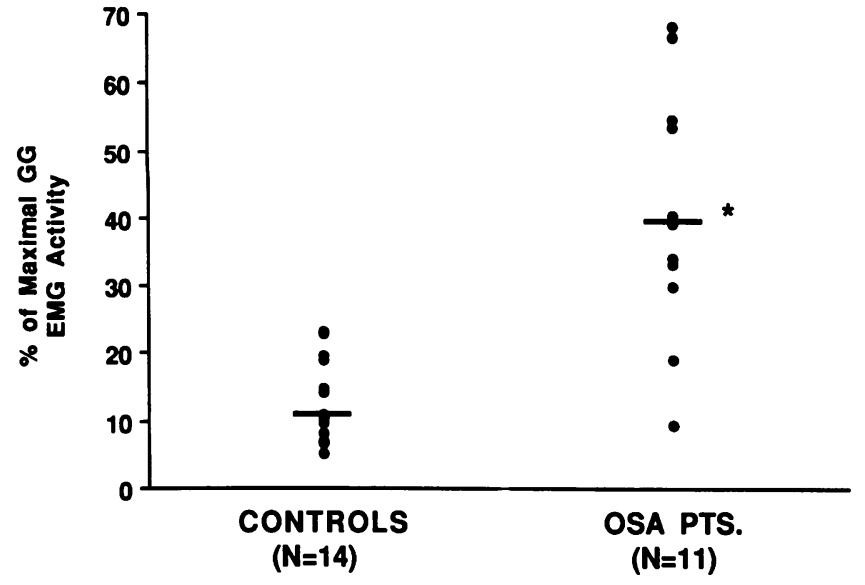

Figure 3. Peak phasic GG EMG activity. Cumulative data from all subjects and patients demonstrating that in the basal state, the genioglossus functions at a higher percentage of maximum in OSA patients than controls. ${ }^{*} P<0.05$ vs. controls.

trols matched for age and body mass index (Table II). As demonstrated in Fig. 6, there is again a highly significant difference in basal GG EMG activity expressed as a percentage of maximum in these two groups (apnea patients $40.2 \pm 6.7 \mathrm{vs}$. controls 7.8 \pm 0.7$)$.

GG EMG activity during nasal CPAP administration. With CPAP administration (Fig. 7), peak phasic GG EMG activity fell significantly in apnea patients but demonstrated little change in the control subjects. In apnea patients, with $5 \mathrm{~cm}$ $\mathrm{H}_{2} \mathrm{O}$ CPAP the EMG fell $46.4 \%$ to a level of $21.6 \%$ of maximum and with $10 \mathrm{~cm} \mathrm{H}_{2} \mathrm{O}$, the EMG fell $48.2 \%$ to a level of $20.1 \%$ of maximum. In the controls, with $5 \mathrm{~cm} \mathrm{H}_{2} \mathrm{O}$ CPAP the EMG only fell $2.1 \%$ to a level of $12.0 \%$ of maximum while with $10 \mathrm{~cm} \mathrm{H}_{2} \mathrm{O}$ there was virtually no change $(0.4 \%$ fall) from the basal state. However, as shown, peak phasic activity remained significantly higher in apnea patients than controls even during CPAP administration.

Reproducibility. As can be seen in Fig. 8 some intrasubject variability does exist in our genioglossal activity measurement. However, the mean GG activity value for all subjects studied during trials 1 and 2 was not different $(25.1 \pm 7.0$ vs. $21.1 \pm 8.3)$. In addition, the intraclass correlation coefficient is 0.77 indicating very good to excellent agreement for our measurements

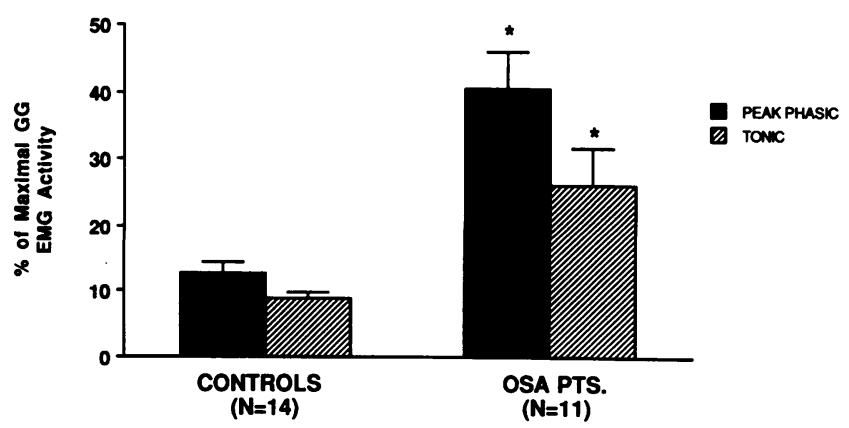

Figure 4. Peak phasic and tonic GG EMG activity. Mean data from all subjects and patients demonstrating that both peak phasic and tonic genioglossal EMG activity was higher in apnea patients compared to controls. ${ }^{*} P<0.05$ vs. controls.

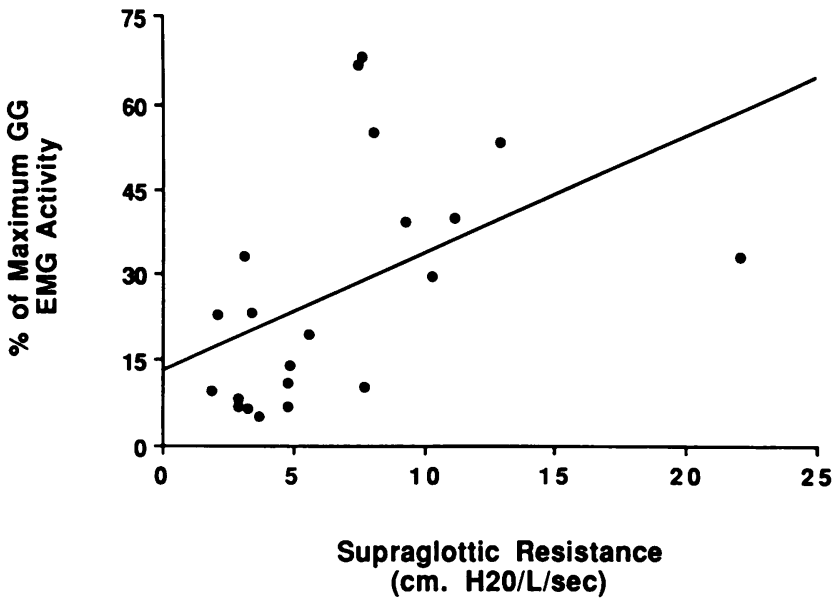

Figure 5. Correlation between supraglottic resistance and GG EMG activity. Linear regression analysis of all subjects demonstrating a significant correlation between supraglottic resistance (measured at 0.2 liter/s inspiratory flow) and the basal peak phasic genioglossal EMG (expressed as a percentage of maximum). $r=0.48 ; P=0.03$.

(35). We believe this supports the reproducibility and validity of our technique.

\section{Discussion}

The principal observations in this study are (a) that GG EMG activity is higher during wakefulness in apnea patients than age- and size-matched controls assumedly in response to diminished airway patency, $(b)$ that despite this increased muscle activity, supraglottic resistance remains higher in the apnea patients, and $(c)$ that CPAP, which delivers positive pressure to the airway, markedly inhibits genioglossal EMG activity in apnea patients but has little effect in control subjects. This decrement in muscle activity in apnea patients likely represents inhibition of a neuromuscular reflex designed to preserve airway patency. Furthermore, in this report we describe a reproducible new technique for quantifying GG muscle activity which allows comparisons between individuals.

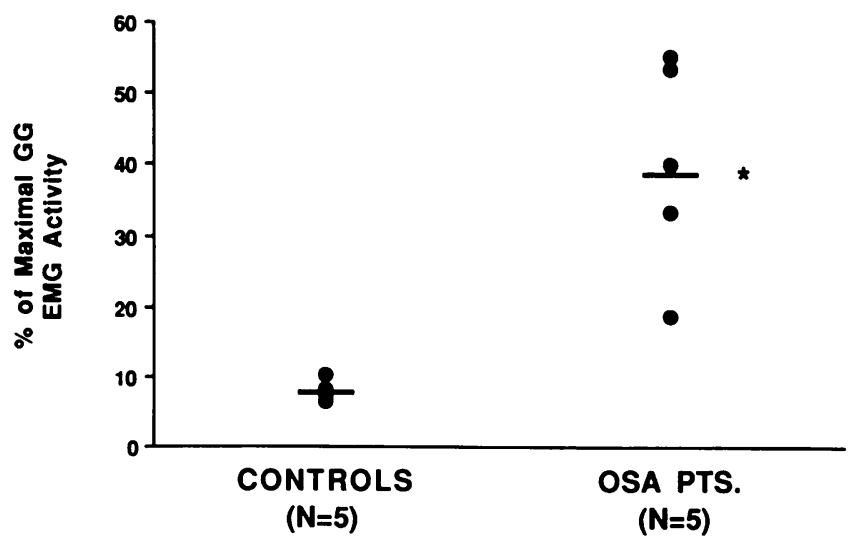

Figure 6. Peak phasic GG EMG activity: age and size-matched data. Cumulative data from five OSA patients and five controls matched for age and body mass index demonstrating that genioglossal activity is higher in apnea patients than controls. ${ }^{*} P<0.05$ vs. controls. 


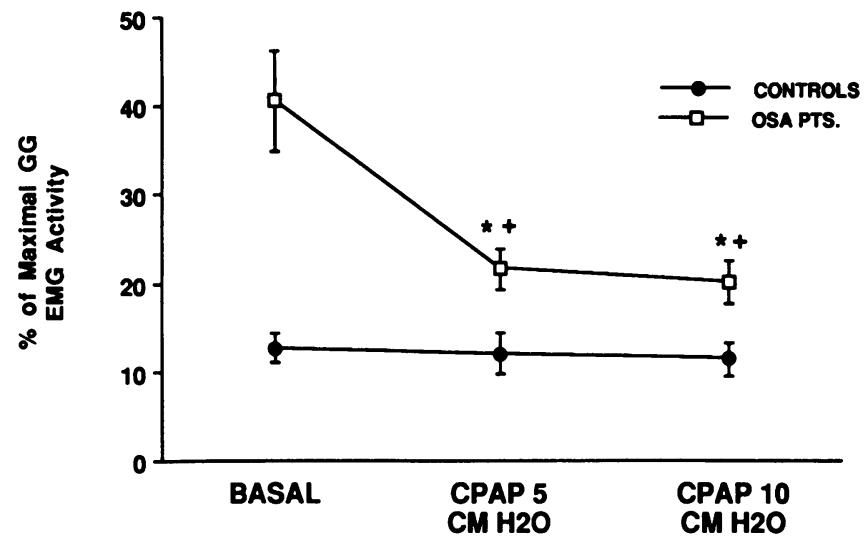

Figure 7. GG EMG activity during nasal CPAP administration. Mean data from all subjects and patients demonstrating that CPAP produces a significant decrement in genioglossal EMG in apnea patients but not controls. ${ }^{*} P<0.05$ vs. controls. $\dagger P<0.05$ vs. basal.

Up till now, comparing GG EMG activity between individuals or groups has proved difficult. As a result, assessment of genioglossal control has been limited to studies of the influence of various states (wakefulness versus sleep) or stimuli on muscle activity. These include sleep states, negative and positive airway pressure, anesthesia, hypercapnia, etc. $(2,3,10,11,32-$ 34, 36-44). Although these studies have provided important information regarding the control of genioglossal activity, these techniques do not allow true comparisons of baseline muscle activity between apnea patients and controls. We have developed the methodology to clearly define a maximum GG EMG in all individuals using both respiratory and nonrespiratory maneuvers. Further, by defining both electrical zero and maximal EMG, we have established a truly quantifiable scale for each individual. Additionally, by the use of the lingual force transducer and negative inspiratory pressure determinations, we can assess individual effort and exclude poor effort as a source of error in these studies. Although this technique is not without potential sources of error (see study limitations), we believe that we can legitimately quantify and compare baseline GG EMG activity between individuals.

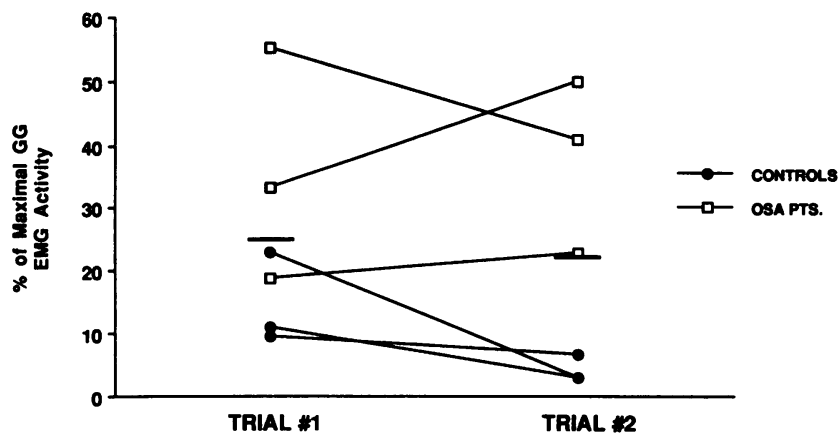

Figure 8. Peak phasic GG EMG activity: reproducibility assessment. Cumulative data from three apnea patients and three controls each studied twice showing variability in individual measurements. Mean GG EMG activity in the six subjects does not change from trial 1 to trial 2 . In addition, the intraclass correlation coefficient was 0.77 indicating good to excellent agreement.
Employing this technique on our two subject populations, we found apnea patients to have a significantly and substantially higher percentage of maximal muscle activity (both peak phasic and tonic) present in the basal state than normal controls (Fig. 3). We believe this increased muscle activity to be a neuromuscular compensatory response to abnormal airway anatomy in an attempt to maintain adequate airway patency. As addressed previously, the preponderance of data indicate that apnea patients have both reduced pharyngeal airway size (5-7) and potentially a more compliant or collapsible airway $(45,46)$. This agrees with our observation of a higher mean supraglottic resistance (Fig. 2) in apnea patients than controls as has been reported previously $(9,46,47)$. This increased resistance occurs despite the augmented GG muscle activity demonstrated in these patients because such a neuromuscular compensatory mechanism is unlikely to be complete and thus not fully restore the airway to normal dimensions or resistance (Fig. 2). On the other hand, this augmented muscle activity may help explain why some authors (9) have been unable to demonstrate clear differences in airway luminal size between apnea patients and age/weight-matched controls when the airway was imaged during wakefulness. These results also agree conceptually with the studies of Suratt et al. (43). Using surface electrodes, they determined the percentage of time that the GG muscle had a phasic signal in apnea patients versus controls. They observed apnea patients to have more phasic activity during both wakefulness and nonobstructed sleep. Because of technical limitations, they could not directly compare muscle activity between groups. However, Suratt et al. (43) did speculate that the GG muscle was probably more active in apnea patients in an attempt to compensate for threatened airway patency.

As shown in Fig. 3, two of the apnea patients demonstrated lower levels of GG muscle activity which clearly overlapped with our normal subjects. We doubt this represented a technical problem as the patients' GG responded to the maximal maneuvers similarly to other subjects. A more likely explanation in these patients is that augmented genioglossal activity was not essential for the maintenance of their airway patency. There are at least two other muscle groups involved in the maintenance of pharyngeal patency: $(a)$ the hyoid muscles (mylohyoid and geniohyoid) which serve to elevate and draw forward the hyoid bone thus maintaining the tongue and other pharyngeal structures away from the posterior pharyngeal wall, and $(b)$ the muscles of the soft palate (including the tensor palatini and palatoglossus) which substantially influence palatal position, route of breathing and airway patency (48). The activity of the palatal muscles seems particularly important as in many OSA patients airway closure occurs behind the palate in the velopharynx while in others, collapse occurs behind the tongue in the hypopharyngeal area $(49,50)$. This seems important in that recent data (51) suggest that the tensor palatini responds preferentially to increases in airway resistance in the immediate vicinity of the palate while the GG activates preferentially to increments in oropharyngeal resistance. Other muscles, like the palatoglossus, may also play an important role in maintaining patent nasal airflow in apnea patients and controls. This raises the possibility that pharyngeal dilator muscles may respond quite specifically during wakefulness depending upon the site of anatomic limitation (velopharynx versus oroor hypopharynx). If this is indeed true, then the varying level of GG activity that we observed in apnea patients may be related very directly to the varying site of anatomic limitation in these 
patients. In addition, in some apnea patients collapse does not occur in the pharynx at all, but in the laryngeal area (52), thus potentially explaining a very low genioglossal activity in some patients.

Both animal and human data support the concept that the genioglossus muscle can respond to increased negative intraluminal airway pressure in a reflex manner. Mathew et al. (12, 13), using the isolated rabbit upper airway, demonstrated that negative pressure applied locally to the upper airway selectively activated upper airway muscles (alae nasi, GG) and positive pressure decreased muscle activity. Furthermore, they showed that anesthetizing the upper airway or denervating afferent output from the airway could inhibit the response of these muscles to negative pressure. Van Lunteren et al. (15) observed a similar selective upper airway muscle response to negative pressure in spontaneously breathing dogs while Issa et al. (16) demonstrated a selective genioglossal response to upper airway occlusion in tracheostomized nonanesthetized dogs. By studying humans in a body plethysmograph Leiter and Dauben speck (19) were able to demonstrate selective activation of the GG compared to the diaphragm in response to increased airway negative pressure suggesting that similar reflex mechanisms are present in the upper airway of humans. Finally, Horner et al. (18) observed in humans that genioglossal muscle activation in response to sudden negative airway pressure occurs with a brief latency (34 ms) consistent with a neural reflex. This may represent a muscle stretch reflex responding to airway deformation although other possibilities exist.

In apnea patients, greater intraluminal negative pressure is generated during inspiration to overcome increased airway resistance. Thus, the augmented genioglossus muscle activity that we have demonstrated may be a reflex response to increased intraluminal negative pressure. This speculation is strengthened by our CPAP results. CPAP, as described by Kuna and Remmers (53), renders upper airway pressure positive rather than negative and, in addition, as suggested by Strohl and Redline (54), may pneumatically splint the pharyngeal airway. Thus, we would expect that reversing intraluminal airway pressure from negative to positive and preventing airway collapse would turn off reflex stimulation of the genioglossus and obviate the need for increased GG muscle activity. As apnea patients have, during normal breathing, greater airway negative pressure as well as a more "collapsible" airway (45, 46), CPAP would be expected to have a greater impact upon apnea patients than controls. This is clearly the case as is shown in Figure 7. These results are consistent with the findings of both Strohl and Redline (54) and Kuna et al. (39) who observed marked reductions in upper airway muscle activity in response to nasal CPAP (GG and alae nasi) in OSA patients. However, negative intraluminal pressure is unlikely to explain the augmented tonic GG muscle activity encountered in apnea patients (Fig. 4) as airway pressure is positive during this respiratory phase. In addition, CPAP pressures up to $10 \mathrm{~cm} \mathrm{H}_{2} \mathrm{O}$ did not completely return even peak phasic GG EMG to the levels encountered in the controls (Fig. 7). Therefore, we would have to speculate that additional sources of muscle stimulation must exist in the pharyngeal airway of apnea patients which may relate to arterial chemistry or pharyngeal receptors sensitive to nonpressure stimuli.

This observed neuromuscular compensation in the GG muscle of apnea patients during wakefulness may help explain the observation of others $(1,2)$ that genioglossal activity falls in
OSA patients during sleep. As discussed previously, many studies indicate that respiratory neuromuscular responses (cough, inspiratory load compensation, chemoresponses) are diminished or lost during sleep. Therefore, it would not be surprising if the mechanisms required to augment genioglossal activity in apnea patients are also markedly attenuated during sleep leading to decreased muscle activity and subsequently airway collapse. Because such mechanisms are not active in normal subjects, genioglossal activity is little affected by sleep in these individuals.

Several methodological considerations must be addressed when assessing our conclusions. First, the relationship between electrical activity (EMG) and muscle shortening or contraction is complex and cannot, in the human upper airway, be directly assessed. Thus, there is no way to confirm that the increased EMG activity observed in apnea patients does in fact correlate with increased muscle force and the expected physiologic outcome. However, our ability to demonstrate a clear relationship between maximal muscle contractile maneuvers, both respiratory (maximum inspiratory pressure) and nonrespiratory (lingual force), with maximal EMG suggests that muscle EMG and performance are closely linked and our observations are therefore valid. Second, our technique of defining basal EMG as a percentage of maximum (between electrical zero and the maximum voluntary signal) is only consistently valid if electrical noise is minimal and similar in all subjects. Substantial background noise would yield a higher basal signal in that individual. Differences in background noise may have accounted for some of the intrasubject differences in GG EMG activity that we demonstrated (Fig. 8). However, we doubt this represents an important problem as there is no reason to believe that greater electrical noise exists in apnea patients compared to controls. In addition, when CPAP was applied the EMG fell substantially in the apnea patients, again suggesting that noise was not the cause of the increased muscle activity observed in these patients. Third, we could not guarantee that recording wire placement was precisely the same in all participants. Differences in wire position may explain some of the variability seen in an individual subject from one study to the next although we believe our reproducibility was quite acceptable. Fourth, differences in negative inspiratory pressure between apnea patients and controls were observed (Table III). There is the possibility that the apnea patients were not giving similar levels of effort and therefore the maximum GG EMG was falsely low (making basal activity a falsely high percentage). However, as noted in the techniques section, maximum inspiratory effort against an occluded airway produced the sole maximum GG EMG in only $8 \%$ of all subjects (one apnea patient and one control). On the other hand, apnea patients tended to produce similar to greater force against the lingual transducer when compared to controls. In that as maximal EMG was generally defined by this tongue protrusion maneuver, it seems unlikely that varying effort influenced our results. Fifth, we did not perform clinical sleep studies on all of our control subjects including two of the age- and size-matched controls. As a result, it is possible that some of these individuals actually had the OSA syndrome. However, it has been demonstrated (55) that historical screening in low risk subjects is very effective in ruling out OSA. Also, many of the normal subjects involved in this study had participated in other research projects in our lab that actually involved some sleep monitoring. None of the subjects so studied demonstrated any sleep-disordered breathing. 
Therefore, we doubt that any of our controls had any substantial disordered breathing during sleep. Finally, although our control subjects were age-matched to the patients, most were not matched for body mass index. This represented a prospective decision not to attempt matching for weight or body mass index because we wished to compare apnea patients to truly normal age-matched controls and not obese normal subjects. However, for the subset of subjects that were weight-matched to apnea patients, our comparisons remain valid. Thus, it appears that factors other than weight are important in determining GG muscle activity.

In conclusion, we have developed new methodology for quantitatively assessing GG muscle activity. Using this technique we have observed a marked increase in GG activity in the basal unstimulated state in apnea patients compared to age-matched controls. We have also demonstrated a significant decrement in this augmented muscle activity with the application of positive airway pressure. We believe that this decrement in muscle activity is due to the inhibition of a neuromuscular reflex designed to preserve airway patency. We further speculate that sleep may also inhibit this reflex and therefore allow airway collapse in apnea patients. However, further investigation is needed to clearly define the effect of sleep upon this augmented GG muscle activity in apnea patients.

\section{Acknowledgments}

The authors thank Anne M. Rannels for her technical support and Susan Y. Hansen for manuscript preparation.

This work was supported in part by Department of Veterans Affairs Merit Review Funding, a Biomedical Research Support Grant from the University of Colorado Health Sciences Center, and National Institutes of Health Research Training Grant HL-07085.

\section{References}

1. Onal, E., M. Lopata, and T. O'Connor. 1982. Pathogenesis of apnea in hypersomnolence sleep apnea syndrome. Am. Rev. Respir. Dis. 125:167.

2. Remmers, J. E., W. J. DeGroot, E. K. Sauerland, and A. M. Anch. 1978. Pathogenesis of upper airway occlusion during sleep. J. Appl. Physiol. 44:931938.

3. Sauerland, E. K., and R. M. Harper. 1976. The human tongue during sleep: electromyographic activity of the genioglossus muscle. Exp. Neurol. 51:160-170.

4. Strohl, K. P., and L. G. Olson. 1987. Concerning the importance of pharyngeal muscles in the maintenance of upper airway patency during sleep. Chest. 92:918-920.

5. Haponik, E., P. Smith, M. Bohlman, R. Allen, S. Goldman, and E. Bleecker. 1983. Computerized tomography in obstructive sleep apnea: correlation of airway size with physiology during sleep and wakefulness. Am. Rev. Respir. Dis. 127:221-226.

6. Rivilin, J., V. Hoffstein, J. Kalbfleisch, W. McNicholas, N. Zamel, and A. Bryan. 1984. Upper airway morphology in patients with idiopathic obstructive sleep apnea. Am. Rev. Respir. Dis. 129:355-360.

7. Suratt, P., P. Dee, R. Attkinson, P. Armstrong, and S. Wilhoit. 1983. Fluoroscopic and computed tomographic features of the pharyngeal airway in obstructive sleep apnea. Am. Rev. Respir. Dis. 127:487-492.

8. Bradley, D., I. Brown, R. Grossman, N. Zamel, E. Phillipson, and V. Hoffstein. 1986. Pharyngeal size in snorers, nonsnorers, and patients with obstructive sleep apnea. $N$. Engl. J. Med. 315:1327-1331.

9. Stauffer, J., C. Zwillich, R. Cadieux, E. Bixler, A. Kales, L. Varano, and D. White. 1987. Pharyngeal size and resistance in obstructive sleep apnea. Am. Rev. Respir. Dis. 136:623-627.

10. Sauerland, E. K., W. C. Orr, and L. E. Hairston. 1981. EMG patterns of oropharyngeal muscles during respiration in wakefulness and sleep. Electromyogr. Clin. Neurophysiol. 21:307-316.

11. Wiegand, L., C. Zwillich, D. Wiegand, and D. P. White. 1989. Collapsibility of the human upper airway during normal sleep. J. Appl. Physiol. 66:18001808.
12. Mathew, O. P., Y. K. Abu-Osba, and B. T. Thach. 1982. Influence of upper airway pressure changes on genioglossus muscle respiratory activity. $J$. Appl. Physiol. 52:438-444.

13. Mathew, O. P., Y. K. Abu-Osba, and B. T. Thach. 1982. Genioglossus muscle responses to upper airway pressure change: afferent pathways. J. Appl. Physiol. 52:445-450.

14. Van Lunteren, E., and K. P. Strohl. 1986. The muscles of the upper airway. Clin. Chest Med. 7:171-188.

15. Van Lunteren, E., W. B. VandeGraff, D. M. Parker, J. Mitra, M. A. Haxbiu, K. P. Strohl, and N. S. Cherniack. 1984. Nasal and laryngeal reflex responses to negative upper airway pressure. J. Appl. Physiol. 56:746-752.

16. Issa, F. G., P. Edwards, E. Szeto, D. Lauff, and C. Sullivan. 1988. Genioglossus and breathing responses to airway occlusion: Effect of sleep and route of occlusion. J. Appl. Physiol. 64:543-549.

17. Basner, R. C., P. M. Simon, R. M. Schwartzstein, S. E. Weinberger, and J. W. Weiss. 1989. Breathing route influence upper airway muscle activity in awake normal adults. J. Appl. Physiol. 66:1766-1771.

18. Horner, R. L., J. A. Innes, K. Murphy, and A. Guz. Evidence for reflex upper airway dilator muscle activation by sudden negative airway pressure in man. 1991. J. Physiol. (Lond.). 436:15-29.

19. Leiter, J. C., and J. A. Daubenspeck. 1990. Selective reflex activation of the genioglossus in humans. J. Appl. Physiol. 68:2581-2587.

20. Iber, C., A. Berssenbrugge, J. Skatrud, and J. A. Dempsey. 1982. Ventilatory adaptations to resistive loading during wakefulness and non-rem sleep. $J$. Appl. Physiol. 52:607-614.

21. Weigand, L., C. W. Zwillich, and D. P. White. 1988. Sleep and the ventilatory response to resistive loading in normal man. J. Appl. Physiol. 64:1186-1195.

22. Jamal, K., G. McMahon, G. Edgell, and J. A. Fleetham. 1983. Cough and arousal responses to inhaled citric acid in sleeping humans. Am. Rev. Respir. Dis. 127:A237. (Abstr.)

23. Power, J. T., C. Stewart, J. J. Connaughton, H. M. Brash, C. M. Shapiro, D. C. Flenley, and N. J. Douglas. 1984. Nocturnal cough in patients with chronic bronchitis and emphysema. Am. Rev. Respir. Dis. 130:999-1001.

24. Berthon-Jones, M., and C. E. Sullivan. 1982. Ventilatory and arousal responses to hypoxia in sleeping humans. Am. Rev. Respir. Dis. 125:632-639.

25. Berthon-Jones, M., and C. E. Sullivan. 1984. Ventilatory and arousal responses to hypercapnia in normal sleeping adults. J. Appl. Physiol. 57:59-67.

26. Douglas, N. J., D. P. White, J. V. Weil, C. K. Pickett, R. J. Martin, D. W. Hudgel, and C. W. Zwillich. 1982. Hypoxic ventilatory response decreases during sleep in man. Am. Rev. Respir. Dis. 125:286-289.

27. Douglas, N. J., D. P. White, C. K. Pickett, J. V. Weil, and C. W. Zwillich. 1982. Hypercapnic ventilatory response in sleeping adults. Am. Rev. Respir. Dis. 126:758-762.

28. White, D. P., N. J. Douglas, C. K. Pickett, J. V. Weil, and C. W. Zwillich. 1982. Hypoxic ventilatory responses during sleep in normal premenopausal women. Am. Rev. Respir. Dis. 126:530-533.

29. Rechtschaffen, A., and A. Kales. 1968. A Manual of Standardized Terminology, Techniques, and Scoring System for Sleep Stages of Human Subjects. (NIH Publ. No. 204). Brain Information Service/Brain Research Institute, University of California, Los Angeles.

30. Barlow, S., and J. Abbs. 1983. Force transducers for the evaluation of labial, lingual, and mandibular motor impairment. J. Speech Hearing Res. 26:616-621.

31. Dworkin, J. 1980. Tongue force in normals and in dysarthric patients with amyotrophic lateral sclerosis. J. Speech Hearing Res. 23:828-837.

32. Krol, R. C., S. L. Knuth, and D. Bartlett, Jr. 1984. Selective reduction of genioglossal muscle activity by alcohol in normal human subjects. Am. Rev. Respir. Dis. 129:247-250.

33. Leiter, J. C., S. L. Knuth, and D. Bartlett, Jr. 1985. The effect of sleep deprivation on activity of the genioglossus muscle. Am. Rev. Respir. Dis. 132:1242-1245

34. Redline, S. and K. P. Strohl. 1987. Influence of upper airway sensory receptors on respiratory muscle activation in humans. J. Appl. Physiol. 63:368374.

35. Fleiss, J. 1981. Statistical Methods for Rates and Proportions. J. Wiley \& Sons, Inc., New York. 211-236.

36. Alex, C. G., R. M. Aronson, E. Oral, and M. Lopata. 1987. Effects of continuous positive airway pressure on upper airway and respiratory muscle activity. J. Appl. Physiol. 6:2026-2030.

37. Hudgel, D. W., M. Mulholland, and C. Hendricks. 1987. Neuromuscular and mechanical responses to inspiratory resistive loading during sleep. J. Appl. Physiol. 63:603-608.

38. Jeffries, B., R. T. Bronouillette, and C. E. Hunt. 1984. Electromyographic study of some accessory muscles of respiration in children with obstructive sleep apnea. Am. Rev. Respir. Dis. 129:696-702.

39. Kuna, S. T., D. G. Bedi, and C. Ryckman. 1988. Effect of nasal airway positive pressure on upper airway size and configuration. Am. Rev. Respir. Dis. 138:969-975.

40. Kuna, S. T., and J. Smickley. 1988. Response of genioglossus muscle 
activity to nasal airway occlusion in normal sleeping adults. J. Appl. Physiol. 64:347-353.

41. Onal, E., M. Lopata, and T. D. O'Connor. 1981. Diaphragmatic and genioglossal electromyogram responses to $\mathrm{CO}_{2}$ rebreathing in humans. J. Appl. Physiol. 50:1052-1055.

42. Patrick, G. B., K. P. Strohl, S. B. Rubin, and M. D. Altose. 1982. Upper airway and diaphragm muscle responses to chemical stimulation and loading. $J$. Appl. Physiol. 53:1133-1137.

43. Suratt, P., R. McTier, and S. Wilhoit. 1988. Upper airway muscle activation in augmented in patients with obstructive sleep apnea compared with that in normal subjects. Am. Rev. Respir. Dis. 137:889-894.

44. White, D. P. 1989. Genioglossal response to respiratory stimuli in apnea patients versus controls. Am. Rev. Respir. Dis. 139:A446.

45. Brown, I. G., T. D. Bradley, E. A. Phillipson, N. Zamel, and V. Hoffstein. 1985. Pharyngeal compliance in snoring subjects with and without obstructive sleep apnea. Am. Rev. Respir. Dis. 132:211-215.

46. Suratt, P. M., R. F. McTier, and S. C. Wilhoit. 1985. Collapsibility of the nasopharyngeal airway in obstructive sleep apnea. Am. Rev. Respir. Dis. 132:967-971.

47. Anch, A. M., J. E. Remmers, and H. Bunce III. 1982. Supraglottic airway resistance in normal subjects and patients with occlusive sleep apnea. J. Appl. Physiol. 53:1158-1163.
48. Rodenstein, D. O., and D. C. Stanescu. 1986. The soft palate and breathing. Am. Rev. Respir. Dis. 134:311-325.

49. Hudgel, D. W. 1986. Variable site of airway narrowing among obstructive sleep apnea patients. J. Appl. Physiol. 61:1403-1409.

50. Shepard, J. W., and S. E. Thawley. 1990. Localization of upper airway collapse during sleep in patients with obstructive sleep apnea. Am. Rev. Respir. Dis. 141:1350-1355.

51. Wasicko, M., J. C. Leiter, J. S. Erlichman, and D. Bartlett. 1990. Reflex control of segmental upper airway resistance during wakefulness in normals and patients with obstructive sleep apnea. Am. Rev. Respir. Dis. 141:A669.

52. Rubinstein, I., A. S. Slutsky, N. Zamel, and V. Hoffstein. 1988. Paradoxical glottic narrowing in patients with severe obstructive sleep apnea. J. Clin. Invest. 81:1051-1055.

53. Kuna, S. T., and J. E. Remmers. 1984. Neural and anatomic factors related to upper airway occlusion during sleep. Med. Clin. North Am. 69:12211242.

54. Strohl, K. P., and S. Redline. 1986. Nasal CPAP therapy, upper airway muscle activation, and obstructive sleep apnea. Am. Rev. Respir. Dis. 134:555558.

55. Young, T., D. Zaccarro, R. Leder, K. Paulus, S. Weber, J. Skatrud, S. Badr, and J. Dempsey. 1991. Prevalence and correlates of sleep disordered breathing in the Wisconsin sleep cohort study. Am. Rev. Respir. Dis. 143:A380. 